\title{
An Adaptive Weighted Threshold Image Restoration Method Based on Wavelet Domain
}

\author{
Lili Chen \\ Laboratory of Intelligent Information Processing, Suzhou University, Suzhou 234000, Anhui, China \\ Hongjun Guo* \\ Laboratory of Intelligent Information Processing, Suzhou University, Suzhou 234000, Anhui, China. \\ *Corresponding author
}

Received: September 3, 2020. Revised: February 28, 2021. Accepted: April 6, 2021. Published: April 8, 2021.

\begin{abstract}
Due to the limitation of imaging equipment, the influence of transmission medium and external environment, image quality degradation will inevitably occur in the process of generation, transmission and reception. These degradation not only worsens the visual effect of the image, but also makes the image lose a lot of useful information, which seriously affects image recognition, target detection and other high-level visual analysis. Wavelet analysis can extract useful information from image signal and meanwhile its profound wavelet basis can get adapted to signals of different properties. To better apply wavelet transform into image restoration domain, this paper according to the characteristics of wavelet transform, analyzes the method to select threshold function and the relationship within and between layers of wavelet coefficients, gets a proper threshold weight coefficient and propose an adaptive weighted threshold image restoration method based on wavelet domain, which makes smaller deviation and variance between the de-noised image and the original signal. The experiment result shows that the algorithm of this paper can obtain good subjective and objective image quality and effectively retain most detailed information of the image.
\end{abstract}

Keywords - Image Restoration, Wavelet Analysis, Threshold Function.

\section{INTRODUCTION}

$\mathrm{I}^{\mathrm{n}}$ $\mathrm{n}$ the actual process, due to the existence of noise, defocus and relative motion and other factors, the image may be blurred. For this reason, researchers on how to make the blurred image more clear to meet people's effective capture of target information. Image restoration is the inverse process of degradation, reducing noise, blur, distortion and other phenomena. The purpose of image restoration is to recover the high-quality image which is close to the original image from the observed degraded image [1]. Traditional inverse filtering methods often lose a lot of high-frequency information such as texture and edge, which can not meet the application requirements. Therefore, it is very important to improve the quality of image restoration algorithm. Besides, with the swift development of artificial intelligence (AI) technology, the acquisition of image also facilitates the carry-forward of the research and development of the latest technology such as machine film-reading [2]. Wavelet analysis is a newly-emerging mathematical branch formed in the late 1980s. It includes rich mathematical content and promotes the theoretical development of functional analysis and harmonic analysis; in the meanwhile, it has been widely applied in the fields like signal de-noising, image compression and adaptive filtering and it is the hot spot of many disciplines. Wavelet analysis is a milestone in the development history of Fourier analysis and harmonic analysis and it is known as "mathematical microscope". As a multi-resolution analysis method, wavelet analysis has an excellent time-frequency localization characteristic and it is particularly suitable for the image coding method designed according to the visual characteristics of human eyes, but also good for the progressive transmission of image signals [3] [4]. In practical applications, the image coding based on wavelet transform is better than conventional methods in both image de-noising and restoration quality. The proposal of adaptive wavelet transform has injected new vigor into wavelet image processing. Compared with conventional wavelet transform, it doesn't rely on Fourier transform and it has the strengths of simple computation, and low time and spatial complexity. In the field of image processing, it has been an important issue of study to get the original image from the incomplete, indirect and noisy image [5]. When acquiring an image, the noises from sensors and channel transmission have also been added to the image. Due to the existence of noises, the image has an appearance of mottling, graininess or snowflake or redundant texture. So, the image is not only fuzzy in the outlook, but further image processing is also affected. This study has made improvements on image de-noising and restoration method based on adaptive wavelet.

Researchers have paid close attention to how to obtain approximately original image from the noisy degraded image. Image restoration can be deemed as the process to tell noises from information of the original image in a degraded image, then to remove the noise information and to preserve as much information of the original image as possible [6]. Image 
de-noising can be divided into spatial-domain method and transform-domain method. The latter transform the image from the spatial domain to another domain, e.g. frequency domain and spatial domain and then de-noise it in the transform domain. The former is to conduct de-noising in the spatial domain [7]. Mathematically, Mallat, Choen, Lawton and Daubechiese and others have made major contributions to the construction and performance of wavelet; in particular, they have constructed compact-supported orthogonal wavelet with multi-resolution analysis theories and vanishing moment conditions; however, the wavelet and scale function constructed are not relevant to the signal analyzed. In this case, when analyzing the signals, the selection of wavelet basis is usually subjective and relies on plenty of analysis and summary of experiments [8]. So some scholars have studied to search and analyze the most similar to the signal from the wavelet library obtained or to get an improved form of the existing wavelet. Some other scholars, including Chapa and Rao, have constructed the corresponding wavelet and scale function from the given signals and then analyze and process the signals with the wavelet obtained. On one hand, it has overcome the difficulty of hose the given signal selects the proper wavelet and on the other, the result of signal analysis can better reflect the features of signal. Regarding image de-noising and restoration, wavelet transform has made a great success due to its low entropy, de-relevance and flexibility to choose the basis [9]. However, only Haar function is the symmetric compact-supported scale function and other scale functions can't meet these requirements. But in signal processing, it requires wavelet scale function to have these excellent characteristics. Therefore, adaptive wavelet is constructed.

This paper firstly has analyzed image restoration and wavelet threshold function as well as the characteristics of wavelet transform. Then it has mainly discussed the method of using adaptive wavelet transform to perform image threshold de-noising. According to the characteristics of adaptive wavelet transform and the image features, it has also studied the image restoration method with adaptive wavelet. Besides, it has also analyzed the selection method and importance of threshold function. Finally, the test experiment has proven that the algorithm in this paper is effective.

\section{II.IMAGE DEGRADATION}

\section{A. Estimation of degradation function}

Assume that the original image is $f(x, y)$, then the degraded image can be represented as

$$
g(x, y)=H[f(x, y)]+\eta(x, y)
$$

Where $H$ is the degradation function and $\eta(x, y)$ is the additive noise. The goal of restoration is to obtain an estimate $\hat{f}(x, y)$ based on degraded image with a view to making the image as close to the original image as possible.
If $H$ is a linear and spatially invariant space, then the degraded image can be represented as follows in the space frequency domain.

$$
g(x, y)=h(x, y) * f(x, y)+\eta(x, y)
$$

After Fourier transform, below can be obtained

$$
G(u, v)=H(u, v) F(u, v)+N(u, v)
$$

There are many kinds of noise models, including Gaussian noise, salt \& pepper noise, and Poisson noise, etc.

In image restoration, the degradation function needs to be estimated, mainly by the observation method, the experiment method and the mathematical modeling method.

Observation method gets $H(u, v)$ by selecting the sub-image with fewer noises (to reduce the impact of noises) based on which it constructs the $H(u, v)$ of the entire image, and then performs restoration with the subsequent restoration methods. Experiment method means to use or design a device (process) similar to the image degradation to make its imaging into a pulse and then gets the impulse response $H(u, v)=G(u, v) / A$ of the degradation system. And the modeling estimation is to deduce from the basic principles which cause image degradation and then simulate the original image. During the simulation, it adjusts the model parameters to obtain the most accurate degradation model as possible. There are two kinds of models: atmospheric turbulence model and motion blur model.

(1) Atmospheric turbulence model

The universal form is $H(u, v)=e^{-k\left(u^{2}+v^{2}\right)^{5 / 6}}$, where $k$ is a constant related to the property of turbulence. The bigger $k$, the more blurred the image and it has the same form as Gaussian low-pass filter. While achieving this filtering, attention shall be paid to that $u$ and $v$ shall replace their differences with the frequency center respectively due to centralization. Assume that the frequency center is $(\mathrm{M} / 2, \mathrm{~N} / 2)$, then they are replaced as $\mathrm{u}-\mathrm{M} / 2$ and v-N/2 respectively. It is the same as how to realize the previous frequency filter. Its Fourier spectrum is as follows Fig.1.

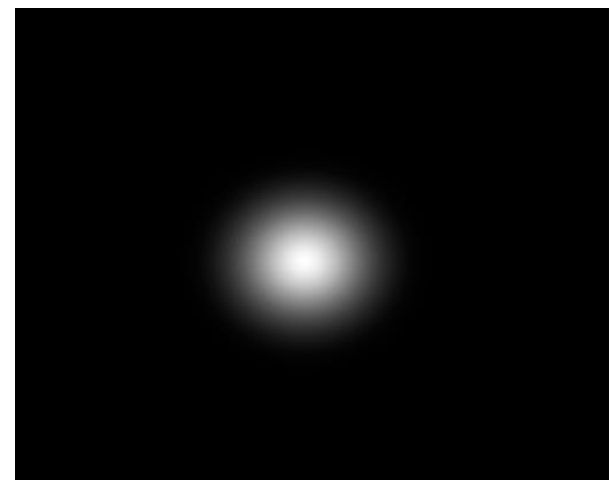

Fig. 1. Fourier spectrum of atmospheric turbulence model 
(2) Motion blur model

The degradation function is

$$
H(u, v)=\frac{T \sin [\pi(u a+v b)]}{\pi(u a+v b)} e^{-j \pi(u a+v b)}
$$

Where $T$ represents the exposure time, $a$ and $b$ are the amount of movement in the horizontal and vertical directions respectively. Please note that $\lim _{x \rightarrow 0} \frac{\sin x}{x}=1$. When $\pi(u a+v b)=0, H(u, v)=T$. In the same way, when achieving this filter, $u$ and $v$ need to be replaced by $\mathrm{u}-\mathrm{M} / 2$ and $\mathrm{v}-\mathrm{N} / 2$ respectively because of centralization.

When $a=b=0.1$ and $T=1$, its Fourier spectrum is as below Fig.2.

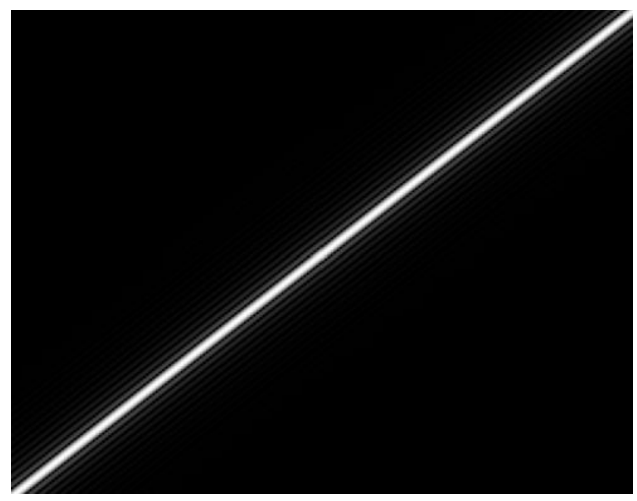

Fig. 2. Fourier spectrum of motion blur model

\section{B. Key image restoration methods}

(1) Direct Inverse Filtering

With the knowledge of the degradation model of the image, the simplest restoration method is inverse filtering. The computation is as follows:

$$
\begin{aligned}
\hat{F}(u, v) & =\frac{G(u, v)}{H(u, v)} \\
& =F(u, v)+\frac{N(u, v)}{H(u, v)}
\end{aligned}
$$

But when $H(u, v)$ is 0 or is close to $0, N(u, v) / H(u, v)$ will become big enough to support the key part of the entire image. Even if $N(u, v)$ is very small or $0, F(u, v)$ will become very big in the computation when $H(u, v)$ is close to 0 . In this way, the restored image has no information.

$H(u, v)$ can be modified by the following two methods:

(1) use a low-pass filter on $G(u, v) / H(u, v)$ to filter the useless high-frequency part (namely to filter the part in which $H(u, v)$ is close to 0$)$;
(2) regulate a value to make $1 / H(u, v)=0$ when $|H(u, v)| \leq \delta$. Both methods can remove or weaken the influence of $H(u, v)$.

(2)Wiener filter (the filter with the lease mean square error)

Wiener filter is a linear image restoration method and it searches an estimation $\hat{f}$ which can minimize the statistical error function $e^{2}=E\left\{(f-\hat{f})^{2}\right\}$ and in the frequency domain, it can be represented as follows

$$
\hat{F}(u, v)=\left\lfloor\frac{1}{H(u, v)} \frac{|H(u, v)|^{2}}{|H(u, v)|^{2}+S_{\eta}(u, v) / S_{f}(u, v)}\right\rfloor G(u, v)
$$

Where $H(u, v)$ is the degradation function; and $S_{\eta}$ and $S_{f}$ are the power spectrums of noises and undegraded image. The signal-to-noise ration (SNR) is

$$
S N R=\frac{S_{f}(u, v)}{S_{\eta}(u, v)}
$$

When the SNR is infinite, it means that there is no noise and the Wiener filter is degraded into inverse filter.

(3) Constrained least square filter

The core of constrained least square filter is H's sensibility to noises. One method to handle this problem is the optimality based on measurement of smoothness. In the restoration, it needs to search the minimum value of criterion function $C$, which is defined as

$$
C=\sum_{x=0}^{M-1} \sum_{y=0}^{N-1}\left[\nabla^{2} f(x, y)\right]^{2}
$$

The constraint of the function is

$$
\|g-H \hat{f}\|=\|\eta\|^{2}
$$

The frequency domain solution to the optimization problem is provided in the following formula

$$
\hat{F}(u, v)=\left[\frac{H^{*}(u, v)}{|H(u, v)|^{2}+\gamma|P(u, v)|^{2}}\right] G(u, v)
$$

Where $P(u, v)$ is the Fourier transform of Laplacian operation $p(x, y)$ and

$$
P=\left[\begin{array}{rrr}
0 & 1 & 0 \\
1 & -4 & 1 \\
0 & 1 & 0
\end{array}\right]
$$


There are two unknowns: $\gamma$ and $\|\eta\|^{2}$. If $\|\eta\|^{2}$ which is proportionate to nose power (scalar) is known, $\gamma$ can be obtained through iteration.

\section{IMAGE RESTORATION AND WAVELET THRESHOLD FUNCTION}

\section{A. Image restoration}

Image restoration is a classic issue in image processing. Conventional restoration methods tend to use mean or linear method, with Wiener filtering being used frequently. Nevertheless, it doesn't have good de-noising. Image restoration technique is proposed mainly targeted for the "degradation" in the imaging process and the so-called "degradation" phenomenon mainly refers to the impact of various factors that affect the imaging process, including the defocus of the imaging system, the relative motion between the equipment and the object or the inhernt defects of the equipment, leading to un-ideal image quality [10]. Image restoration shares something common with image enhancement and it means to improve the overall quality of the image; but in contrast to image restoration technology, image enhancement technology focuses on stretching the contrast while the image restoration technology removes the fuzzy part from the image and restore the true color of the image through defuzzing function. It mainly restores or reconstructs the degraded image with certain so-called priori knowledge of the degraded image and from the perspective of restoration process, it can be seen as a reverse process of image degradation. Image restoration first requires proper estimation of the entire process of image degradation, based on which an approximate degraded mathematical model is built. Afterwards, the model needs proper revision in order to compensate the distortion in the degradation process in order to make sure that the image restored approximates the original image and achieves the best optimization of the image. But in the image degradation, both noises and interference exist, which has brought many uncertainties to image restoration. Add Gaussian noise and impulse noise to an image respectively [11] [12]. Display the original image and the noisy image as well as the corresponding histograms, as shown in Fig.3 below.

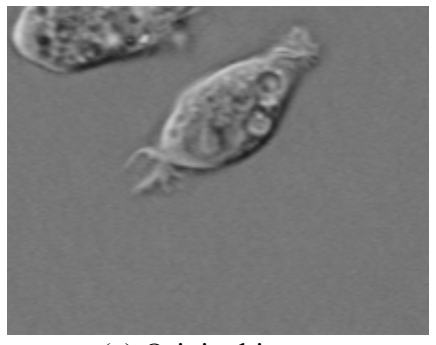

(a) Original image

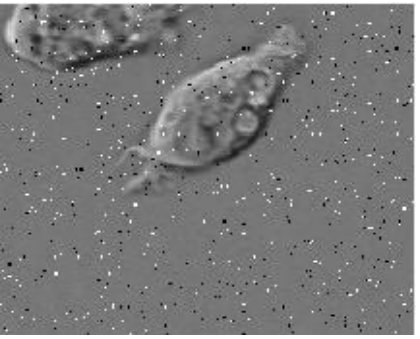

(b) Image with impulse noise

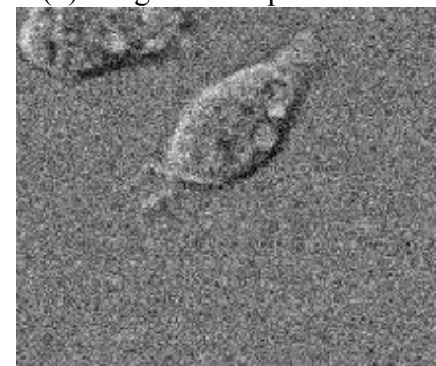

(c) Image with Gaussian noise

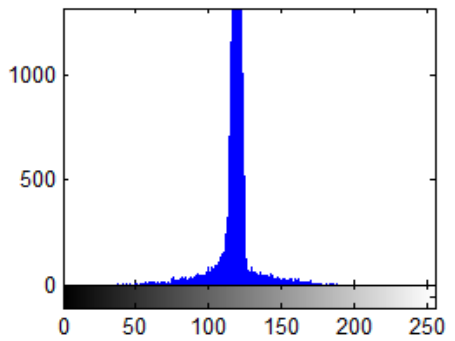

(d) Histogram of original image

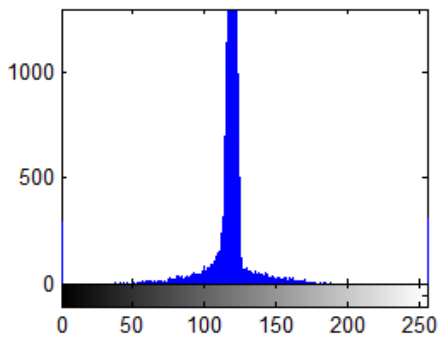

(e)Histogram of image with impulse noise

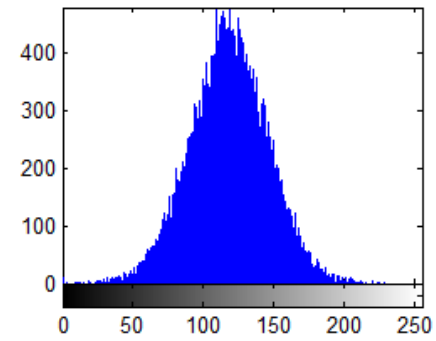

(f) Histogram of image with Gaussian noise

Fig. 3. Impact of noises on image histogram

\section{B. Wavelet function and wavelet variance}

The basic idea of wavelet analysis is to use a cluster of wavelet function system to represent or approximate a certain signal or function. Therefore, wavelet function is the key in wavelet analysis and it refers to the kind of functions which have oscillation and can quickly attenuate to 0 ; in another word, wavelet function $\psi(t) \in L^{2}(R)$ and satisfies the following: 


$$
\int_{-\infty}^{+\infty} \psi(t) d t=0
$$

Where $\psi(t)$ is the wavelet basis function and it can constitute a cluster of function system through scaling and translation in the time axis.

$$
\psi_{a, b}(t)=|a|^{-1 / 2} \psi\left(\frac{t-b}{a}\right) a, b \in R, a \neq 0
$$

Where $\psi_{a, b}(t)$ is the sub-wavelet; $a$ is the scale factor and it reflects the cycle length of wavelet; and $b$ is the translation factor which reflects the translation in time.

It needs to be pointed out that to choose the proper wavelet basis function is the premise for wavelet analysis. In practical application research, the wavelet basis function shall be selected according to the actual circumstance. For the same signal or time sequence, if different wavelet basis functions are selected, they usually have different results and sometimes these results may be greatly different. At present whether a wavelet basis function is good or not is mainly determined by the error between its comparison in processing signals with different wavelet analysis and the theoretical result and as a result, the wavelet basis function needed for such study is therefore selected.

If $\psi_{a, b}(t)$ is the sub-wavelet provided by Formula (13), its Continue Wavelet Transform (CWT) for the given finite energy signal $f(t) \in L^{2}(R)$ is as follows:

$$
W_{f}(a, b)=|a|^{-1 / 2} \int_{R} f(t) \bar{\psi}\left(\frac{t-b}{a}\right) d t
$$

Where $W_{f}(a, b)$ is the wavelet transform coefficient; $f(t)$ is $a$ signal or quadratically integrable function; $a$ is the scaling scale; $b$ is the translation parameter; and $\bar{\psi}\left(\frac{x-b}{a}\right)$ is the complex conjugate function of $\psi\left(\frac{x-b}{a}\right)$. The time sequence data observed in the geonomy are mostly discrete. Assume that the function $f(k \Delta t),(k=1,2, \ldots, N ; \Delta t$ is the sampling interval), then the discrete wavelet transform form of Formula (14)

$$
W_{f}(a, b)=|a|^{-1 / 2} \Delta t \sum_{k=1}^{N} f(k \Delta t) \bar{\psi}\left(\frac{k \Delta t-b}{a}\right)
$$

From Formula (14) or Formula (15), the basic principles of wavelet analysis can be learned: it gets the low- or high-frequency information of the signal by increasing or reducing a, analyzes the general picture or details of the signal, and conduct analysis on the local features in different time scale and space.

In practical research, what matters most is to get the wavelet coefficients from wavelet transform equation and then analyze the features in the time and frequency changes of time sequences with these coefficients.

Conduct integral on the squared value of wavelet coefficients in domain $b$ and get the wavelet variance, i.e.

$$
\operatorname{Var}(a)=\int_{-\infty}^{\infty}\left|W_{f}(a, b)\right|^{2} d b
$$

The process in which wavelet variance changes over a is called wavelet variance map. As shown in Formula (16), it can showcase the energy distribution of signal fluctuation over a; therefore, wavelet variance map can be used to determine the relative intensity under different scale disturbance or the main time scale (i.e. the main cycle) in the signal.

\section{Wavelet threshold function}

With its excellent time and frequency properties, wavelet has drawn more and more attention in image restoration and has pioneered a way of restoring image and removing noises with non-linear methods. The reason why wavelet can restore is that wavelet transform has the sparse distribution of wavelet coefficient which lowers the entropy after image transform. As multi-resolution methods are used, non-stationarity of signals are vividly depicted, including sudden changes or breaking points. Noises can be eliminated according to the distributions of signals and noises in different resolutions. Wavelet transform can decorrelate signals and the noises have the trend of whitening after transform; so wavelet domain is better for de-noising than time domain. Wavelet transform can flexibly select basis function, and it can also select more wavelets and wavelet packets according to the characteristics of signal and the requirements of de-noising. For different occasions, different wavelet basis functions can be selected [13].

Threshold processing function includes soft threshold and hard threshold. Assume that $w$ is the size of wavelet coefficient, $w_{\lambda}$ is the size of wavelet coefficient after a threshold is imposed and $\lambda$ is the threshold.

(1) Hard threshold

When the absolute value of the wavelet coefficient is smaller than the given threshold, make it 0 and when bigger than the threshold, keep it unchanged, i.e.

$$
w_{\lambda}=\left\{\begin{array}{l}
w,|w| \geq \lambda \\
0,|w|<\lambda
\end{array}\right.
$$

(2) Soft threshold

When the absolute value of the wavelet coefficient is smaller than the given threshold, make it 0 and when bigger, subtract the threshold, i.e 


$$
w_{\lambda}=\left\{\begin{array}{l}
{[\operatorname{sign}(w)](|w|-\lambda),|w| \geq \lambda} \\
0,|w|<\lambda
\end{array}\right.
$$

Hard threshold function is discontinuous at point $|w|=\lambda$ and it can easily caused significant Pseudo-Gibbs phenomena in the de-noised image near singular points.

The selection of the threshold is a critical step in wavelet de-noising. In this process, wavelet threshold $\lambda$ plays a decisive role: if the threshold is too small, the wavelet coefficient added with threshold will contain too many noise components to achieve the purpose of de-noising; otherwise, if it is too big, it will remove useful components and cause distortion. Given the complexity of algorithm implementation, most adopt Donoho and Johnstone universal threshold $\delta=\sigma \sqrt{2 \log N}$. Here, $\sigma$ is the standard deviation of noises and $\mathrm{N}$ is the size or length of signal. As the threshold is directly proportional to the square root of the logarithm of the size of different signals, when $\mathrm{N}$ is big, the threshold tends to make all wavelet coefficients 0 . At that moment, the wavelet filter has been reduced to a low-pass filter [14].

\section{IMPLEMENTATION STEPS OF ADAPTIVE WEIGHTED THRESHOLD METHOD BASED ON WAVELET DOMAIN}

In the $1^{\text {st }}$ level to perform orthogonal wavelet decomposition on the image, take $\mathrm{HH}$ part from the wavelet coefficient, and calculate the threshold with the estimated value of its standard deviation $\sigma$. The specific steps are as follows:

(1) Wavelet decomposition of 2D signal: select a wavelet and the layers $N$ of wavelet decomposition and then calculate the decomposition of signal s to the $N$ th layer.

(2) Threshold quantization of high-frequency coefficient: for every layer from 1 to $N$, select a threshold and perform threshold processing on the high-frequency coefficients in this layer.

(3) Initial value provided to the parameter $\alpha$ of threshold function: $\alpha_{1}=0$ and $\alpha_{2}=1$. Make $i=1$ and assume $N$ is the times of iterations.

(4) Perform de-noising processing on the image at the parameters $\alpha_{1}$ and $\alpha_{2}$ and calculate the peak signal to noise ratio respectively $\mathrm{PSNR}_{1}$ and $\mathrm{PSNR}_{2}$ of the de-noised image.

(5) Calculate the midpoint of $\alpha_{1}$ and $\alpha_{2} \alpha_{\text {mid }}=\left(\alpha_{1}+\alpha_{2}\right) / 2$, substitute the threshold function, perform de-noising on the image and calculate the PSNR ${ }_{\text {mid. }}$.

(6) Calculate the differential value $\mathrm{PSNR}_{\text {mid }}$ of PSNR at point $\alpha_{\text {mid }}$ in the function with $\alpha$ as the variable. If PSNR' ${ }_{\text {mid }} \geq 0$, then $\alpha_{1}=\alpha_{\text {mid }}$ and $\mathrm{PSNR}_{1}=\mathrm{PSNR}_{\text {mid; }}$ otherwise, $\alpha_{2}=\alpha_{\text {mid }}$ and $\mathrm{PSNR}_{\text {mid }}=\mathrm{PSNR}_{2}$. Then $\alpha_{\text {mid }}$ is the optimal threshold parameter and the corresponding $\mathrm{PSNR}_{\text {mid }}$ is the maximum peak signal-to-noise ratio.

(7) Reconstruction of 2D wavelet: calculate the wavelet reconstruction of $2 \mathrm{D}$ signal according to the low-frequency coefficient in the $N$ th layer and the various high-frequency coefficients from revised the $1^{\text {st }}$ to the $N$ th layer.

The equation of the threshold function is as follows:

$$
\begin{gathered}
\hat{w}_{j, k}= \\
\left\{\begin{array}{cl}
\operatorname{sign}\left(w_{j, k}\right)\left(1 w_{j, k} \mid-\alpha \lambda+\frac{\alpha(1-\alpha) \lambda}{\exp \left(w_{j, k}\right)}\right), & \left|w_{j, k}\right| \geq \lambda ; \\
0, & \left|w_{j, k}\right|<\lambda .
\end{array}\right.
\end{gathered}
$$

In this equation, $\alpha \in[0,1]$. When $\alpha$ is 0 and 1 respectively. Equation (18) becomes hard threshold method and soft threshold method immediately. For $\alpha$ common $\alpha \in(0,1)$, the coefficient $\hat{w}_{j, k}$ estimated is between the soft and hard threshold methods. Make the value of $\left|w_{j, k}\right|$ between $\left|w_{j, k}\right|-\lambda$ and $\left|w_{j, k}\right|$ and it might make the wavelet coefficient $\hat{w}_{j, k}$ estimated closer to $u_{j, k}$. Based on this idea, factor $\alpha$ in threshold estimate can be seen as an independent variable. Take the de-noising result PSNR as the function of $\alpha$. Seek optimization by the optimization algorithm of narrowing range so that the $\alpha$ can be found which maximizes PSNR and better de-noising can be obtained.

The estimation equation of noise deviation is as follows:

$$
\sigma_{i, j}^{2}=\frac{\operatorname{Median}\left(\left|w_{i, j}\right|\right)}{0.6475}
$$

In narrow window, time resolution is high while frequency resolution is low; and in broad window,time resolution is low and frequency resolution is high. For time-varying non-stationary signals, high frequency is suitable for small window and low frequency for big window.

\section{V.TEST EXPERIMENT AND RESULT ANALYSIS}

To verify the effectiveness of the algorithm in this paper, it has been tested with biological image as example. Considering the computation volume and de-noising effect of the algorithm, the experiment selects dB4 wavelet. As for the selection of decomposition scale, a too high scale will bring about much computation and it is found in experiments that higher decomposition scale doesn't necessarily lead to better de-noising performance. After a second thought, the scale is selected 3, and $\sigma=20$. Compare the de-noising and restoration result with the optimal parameters obtained from the algorithm in this paper with other methods. Fig. 4 and Fig. 5 and Tab. 1 are the comparisons of the results by the algorithm of this paper, Wiener filtering, the least square method, Lucy-Richardson method and the algorithm in this paper on 2 test images. 
INTERNATIONAL JOURNAL OF CIRCUITS, SYSTEMS AND SIGNAL PROCESSING

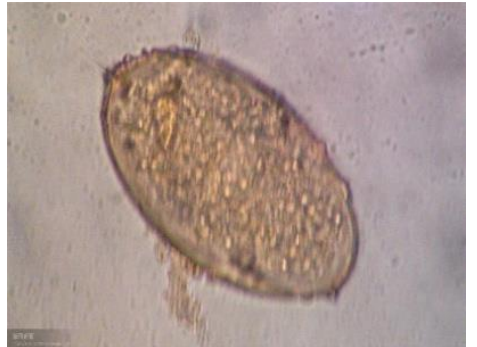

(a)Original image

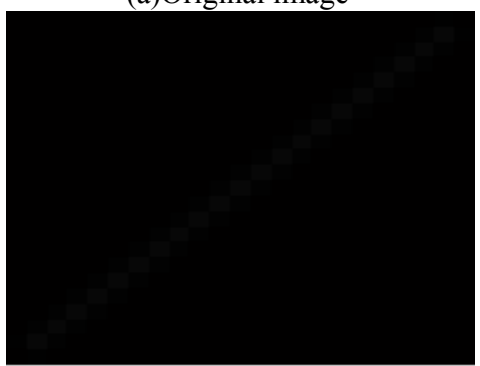

(b) Motion blurred image

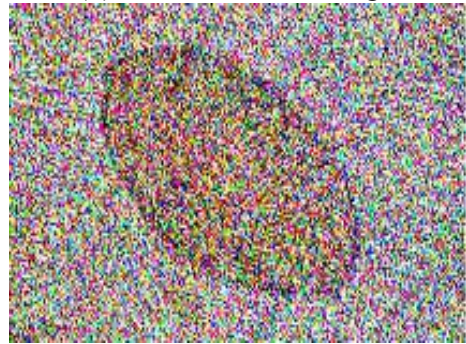

(c) Wiener filtering

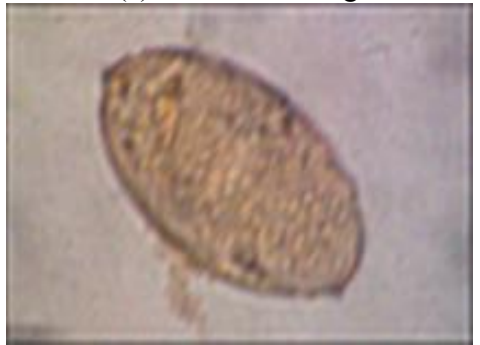

(d) The least square method

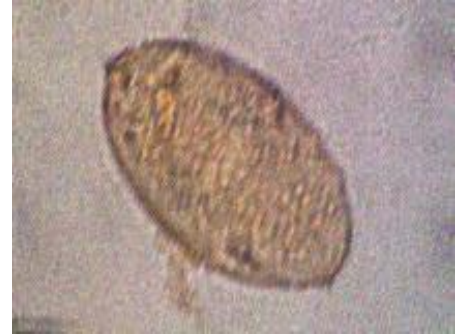

(e) Lucy-Richardson method

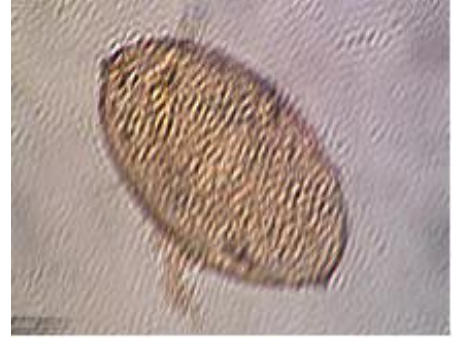

(f) The algorithm in this paper

Fig. 4. Egg of Fasciolabrucelli

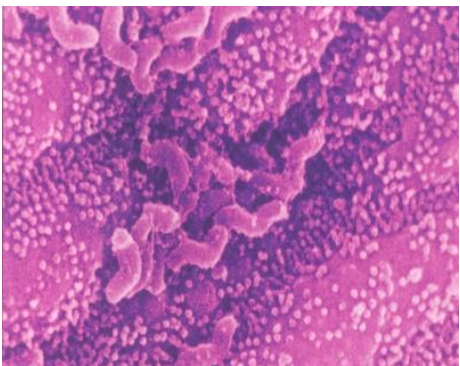

(a)Original image

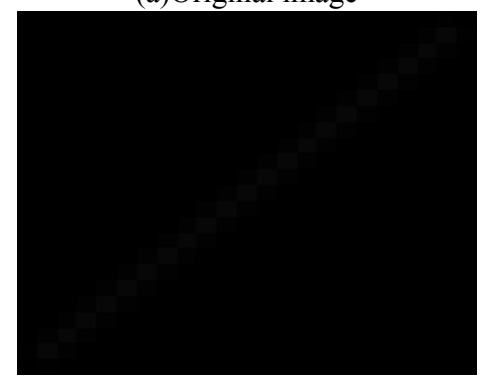

(b) Motion blurred image

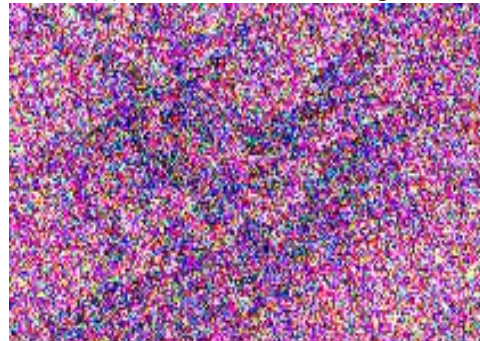

(c) Wiener filtering

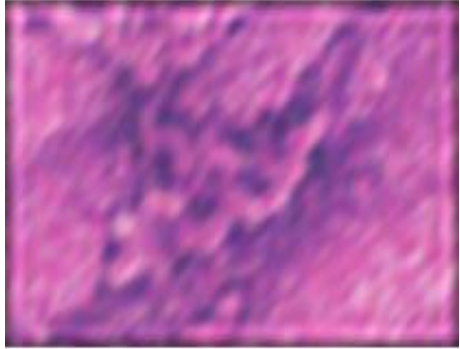

(d) The least square method

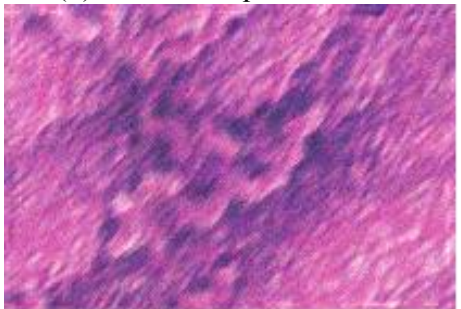

(e) Lucy-Richardson method

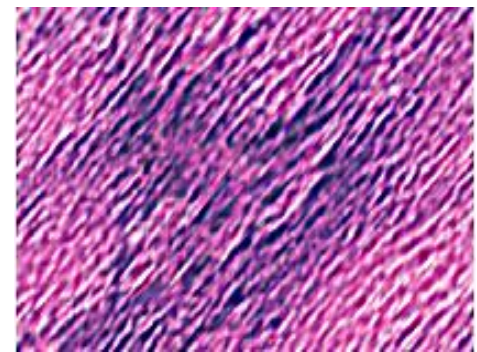

(f) The algorithm in this paper

Fig. 5. Helicobacter pylori on gastric epithelium 
Table 1. PSNR value

\begin{tabular}{|c|c|c|c|c|}
\hline Image & $\begin{array}{c}\text { Wiener } \\
\text { Filter }\end{array}$ & $\begin{array}{c}\text { Least } \\
\text { Square } \\
\text { Method }\end{array}$ & $\begin{array}{c}\text { Lucy-Richar } \\
\text { dson } \\
\text { Method }\end{array}$ & $\begin{array}{c}\text { The } \\
\text { algorithm } \\
\text { in this } \\
\text { paper }\end{array}$ \\
\hline $\begin{array}{c}\text { Egg of } \\
\text { Fasciolabrucelli }\end{array}$ & 37.1523 & 37.7565 & 38.0439 & 38.0872 \\
\hline $\begin{array}{c}\text { Helicobacter } \\
\text { pylori on gastric } \\
\text { epithelium }\end{array}$ & 37.3448 & 37.4081 & 37.7132 & 37.7655 \\
\hline
\end{tabular}

As shown in Tab.1, it can see that the image restoration result has the highest PSNR with the optimal parameter, bigger than all other methods. As indicated in Fig.4 and Fig.5, PSNR is a strong uni-modal function of $\alpha$ with the maximum point which is higher than the hard threshold method when $\alpha=0$ and the soft threshold method when $\alpha=1$. Therefore, midpoint method can be used to find this maximum point. The image restored with this method has a better actual visual effect. It has not only displayed a clear edge contour, but also protected the detail information such as textures. It not only overcomes certain shortcomings of soft and hard threshold methods, but also reaches good restoration effect.

\section{CONCLUSION AND FUTURE WORK}

Wavelet transform is a very effective signal processing method and it can obtain multi-resolution representation of signals; so it has been widely applied in such fields as feature extraction and sparse reconstruction of image information. As wavelet has the following strengths: short support, orthogonality, bi-orthogonality, and symmetry, the selection of wavelet basis restoration and de-noising method has certain superiority. In this paper, we deal with the noise problem of degraded image. The difficulty of degraded image is not only the lack of prior information, but also the problem of noise. Therefore, this paper studies the relevant theoretical knowledge of wavelet transform, and finds that the image can be decomposed into low-frequency and high-frequency bands after wavelet transform. Because most of the noise is concentrated in the high-frequency part, according to this multi-resolution idea, wavelet denoising is mainly carried out in the high-frequency part, while the low-frequency part uses blind restoration method for deblurring operation, which can also reduce the noise. It reduces the computational difficulty and speeds up the computation. In order to keep good edge information and detail features after image denoising, wavelet threshold method is used to denoise by using the time-frequency characteristics of wavelet. Because the commonly used threshold selection method of wavelet adopts global threshold, it ignores that the noise characteristics of different frequency bands are different, so the threshold method is improved. It can adaptively select the threshold for different frequency bands, and then use the adaptive weighted threshold method for restoration. This paper acquire images of true textures and clear details as the goal, has proposed an adaptive weighted threshold image restoration method based on wavelet domain. Besides, it has also combined subjective observation and objective indicators, and comprehensively analyzed the de-noising effect of various methods. As shown in the test experiment, this adaptive wavelet threshold method is feasible and superior.

The future research work is as follows:

(1) We can try the method of deep neural network, and restore the image through the trained network. The deep neural network is mainly used to simulate human brain to learn various abstract features of data, which clearly reflects the essential characteristics of data. According to various learning models, the information is acquired from degraded images for training, and prior information is acquired by learning mechanism and added to the process of image restoration. This can obtain more real prior information, and is also an ideal method. However, the space and time complexity brought by neural network and whether it can effectively solve the practical problems need further research.

(2) However, it is still difficult to evaluate the quality of the restored image. There is no mature and accepted evaluation method available now. Therefore, how to establish a set of mature quality evaluation indicators of restored images will be a direction of future research.

(3) At present, most of the research on the restoration of motion blur image mainly focuses on the motion blur in the whole image. But there is still a local motion blur in reality, that is, the background area is unchanged, only the moving blur is caused by the region through which the target passes. Therefore, how to recover the local motion blur image will be a research direction in the future.

\section{REFERENCE}

[1] Liu, Lin; Wang, Kechao, "Subcellular Structure Segmentation Method in Microscope Image", Acta Microscopica, 2019, vol.28, no.5, pp.1332-1341.

[2] Jiang, Lei; Zhao, Zongying; Zhao, Jianwen, "Medical Tumor Image Classification Algorithms and Its Application in Breast Cancer", Investigacion Clinica, 2019, vol. 60, no. 3, pp.609-618.

[3] C. K. Jha, M. H. Kolekar, "Empirical Mode Decomposition and Wavelet Transform Based ECG Data Compression Scheme", IRBM, In press, corrected proof Available online,30 May, 2020.

[4] Yu, Xiaomo; Li, Wenjing; Wang, Derong, "Agricultural Machinery Autonomous Navigation Scheme Based on Visual Image Processing", Revista De La Facultad De Agronomia De La Universidad Del Zulia, 2019, vol. 36, no. 4, pp.1086-1096.

[5] Ravi Nigam, Sachin K. Singh, "Crack Detection in A Beam Using Wavelet Transform and Photographic Measurements", Structures, 2020, vol.25, no. 6, pp.436-447.

[6] KanikPalodhi, Joydeep Chatterjee, Rupamoy Bhattacharyya, S. Dey, SibajiRaha, "Convolution Based Hybrid Image Processing Technique for Microscopic Images of Etch-Pits in Nuclear Track Detectors", Radiation Measurements, 2020, vol. 130, no. 1, article.106219.

[7] Nimesh Patel, Ashutosh Mishra, "Automated Leukaemia Detection Using Microscopic Images”, Procedia Computer Science, 2015, vol. 58, pp.635-642.

[8] Wen-Bin Shangguan, Guo-Feng Zheng, Subhash Rakheja, Zhihong Yin, "A Method for Editing Multi-Axis Load Spectrums Based on The Wavelet Transforms", Measurement, 2020, vol. 1621, no. 10, pp.107903. 
[9] GuilhermeBeraldi Lucas, Bruno Albuquerque de Castro, Marco Aurélio Rocha, André Luiz Andreoli, "Three-Phase Induction Motor Loading Estimation Based on Wavelet Transform and Low-Cost Piezoelectric Sensors", Measurement, 2020, vol. 164, no. 11, article.107956.

[10] Arvind R. Yadav, R. S. Anand, M. L. Dewal, Sangeeta Gupta, "Gaussian Image Pyramid Based Texture Features for Classification of Microscopic Images of Hardwood Species”, Optik, 2015, vol.126, no .24, pp.5570-5578.

[11] Alexander V. Maltsev, Brian Caffrey, Marta Gonzalez-Freire, Lisa Hartnell, Luigi Ferrucci, "Semi-Automated 3D Segmentation of Human Skeletal Muscle using Focused Ion Beam-Scanning Electron Microscopic Images Reveals Network of Mitochondria", Biophysical Journal, 2020, vol. 118, no. 3, pp.292a-293a.

[12] Yu Wang, Yating Chen, Ningning Yang, Longfei Zheng, Fuqian Shi, "Classification of Mice Hepatic Granuloma Microscopic Images Based on A Deep Convolutional Neural Network", Applied Soft Computing, 2019, vol. 74, no.1, pp.40-50.

[13] Shahrokh Firouzi, Xiangning Wang, "A Comparative Study of Exchange Rates and Order Flow Based on Wavelet Transform Coherence And
Cross Wavelet Transform", Economic Modelling, 2019, vol. 82, no.11, pp.42-56.

[14] Serwan Ali Bamerni, Ahmed Kh. Al-Sulaifanie, "An Efficient Non-Separable Architecture for Haar Wavelet Transform With Lifting Structure", Microprocessors and Microsystems, 2019, vol. 71, no. 11, article.102881.

\section{Creative Commons Attribution License 4.0 (Attribution 4.0 International , CC BY 4.0)}

This article is published under the terms of the Creative Commons Attribution License 4.0 https://creativecommons.org/licenses/by/4.0/deed.en_US 